\title{
Mode-Selective Surface-Enhanced Raman Spectroscopy Using Nanofabricated Plasmonic Dipole Antennas
}

\author{
Weihua Zhang, ${ }^{*, \dagger}$ Holger Fischer,${ }^{\dagger}$ Thomas Schmid, ${ }^{\ddagger}$ Renato Zenobi, ${ }^{\dagger}$ and Olivier J. F. Martin ${ }^{\dagger}$ \\ Nanophotonics and Metrology Laboratory, Swiss Federal Institute of Technology Lausanne (EPFL), \\ 1015 Lausanne, Switzerland, and Department of Chemistry and Applied Biosciences, ETH Zurich, \\ 8093 Zurich, Switzerland
}

Received: May 6, 2009; Revised Manuscript Received: July 2, 2009

\begin{abstract}
Mode-selective surface-enhanced Raman spectroscopy (SERS) is demonstrated using plasmonic dipole antennas fabricated with electron beam lithography. An $\sim 10 \times$ change of the relative enhancement between two different Raman modes is observed when the resonance frequency of the plasmonic antennas is tuned over the Raman modes by varying the geometrical parameters of the antennas, i.e., changing their lengths or narrowing their feeding gaps. The comparison between the Rayleigh scattering spectra and the SERS spectra from the same individual plasmonic dipole antennas indicates that this mode-selective SERS phenomenon is a pure electromagnetic effect, providing a quantitative verification of the electromagnetic mechanism of SERS on a single nanoantenna level.
\end{abstract}

Since the 1970s, it has been observed that the Raman scattering cross-section of molecules can be enhanced for several orders of magnitude when the molecules are adsorbed on a nanostructured noble metal surface. ${ }^{1}$ At the hot spots, such as nanojunctions in colloidal aggregates, the enhancement can even reach $10^{14}$, large enough to extend the sensitivity of this surfaceenhanced Raman spectroscopy (SERS) to the single molecule level, ${ }^{2,3}$ making SERS an ultrasensitive spectroscopic tool. ${ }^{4,5}$ Today, it is widely accepted that the giant enhancement observed in single molecule SERS measurements is mainly caused by the local electric field enhancement, described by the electromagnetic (EM) mechanism of SERS. ${ }^{6}$ Simulations show that the electric field enhancement in the nanojunctions between near-touching nanoparticles can exceed $10,{ }^{2,7}$ enough to make a Raman spectrum from a single molecule detectable. ${ }^{8}$ This has also been proven by experiments, in which a significant increase of Raman enhancement was observed when closing the interparticle gaps. ${ }^{8-11}$ Despite this success, the EM mechanism of SERS still has difficulties in providing an explanation for the phenomena that often accompany the high field enhancement, e.g., variations of Raman spectral profile (i.e., changes of relative intensity between different modes) at different hot spots and the low occurrence of hot spots in colloid aggregates in which there are always many nanogaps. ${ }^{12}$ These issues have stimulated other mechanisms, such as the surface selection rules and the chemical enhancement, related to the adsorption-induced scattering cross-section changes of different vibrational modes. ${ }^{13-15}$ However, it is still not clearly understood as to which mechanism plays a role in SERS and to what exact extent. This is caused by a lack of quantitative methods to evaluate the contribution of each mechanism. It is therefore essential to find a way to investigate the SERS mechanisms quantitatively, in order to build the necessary understanding to implement this technique as a reliable analytical tool.

* Corresponding author. Phone: +41-21-693 3968. Fax: +41-21-693

2614. E-mail: weihua.zhang@epfl.ch.

Swiss Federal Institute of Technology Lausanne (EPFL)

ETH Zurich.

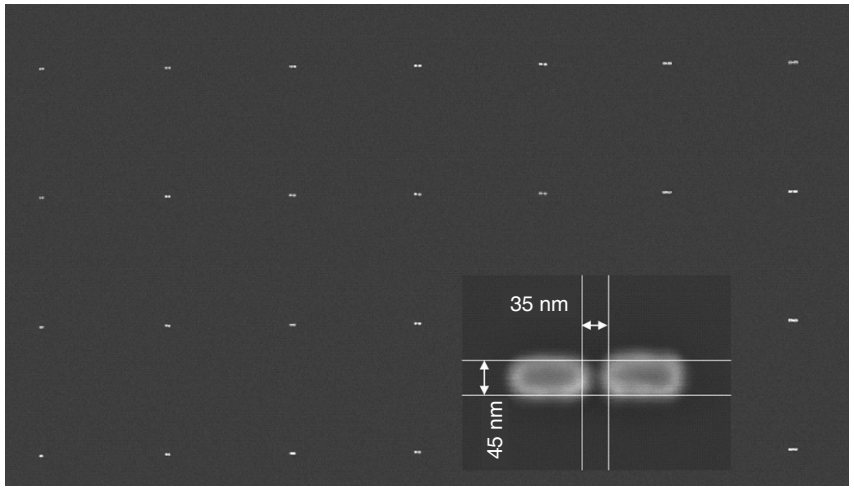

Figure 1. SEM picture of a 2-dimensional antenna array. The inset shows a close view for a typical single plasmonic dipole antenna.

In this work, we use plasmonic dipole antennas to investigate how localized plasmon resonance (LPR) can alter the Raman spectra from nanogaps. As an efficient converter between farfield and near-field signals in the optical regime, a plasmonic dipole antenna provides an ideal model system to investigate the EM mechanism of SERS. It can enhance the electric field by 2 orders of magnitude in the gap at the resonance frequency ${ }^{16,17}$ and can also modify the radiation of a dipole emitter, such as a fluorophore, located in the feeding gap. ${ }^{18}$ Hence, a dipole antenna can influence the two key electromagnetic mechanisms of SERS: the excitation of the molecule and the emission of the Raman signal. Furthermore, the resonance wavelength of a plasmonic dipole antenna can be continuously tuned by changing its geometry. This unique feature enables us to perform a quantitative study of the relationship between LPR and SERS by using the so-called plasmon-sampled-SERS (PS-SERS) technique proposed by van Duyne and co-workers. ${ }^{19}$ In PSSERS, the LPR wavelength of the nanoantenna is tuned over the spectral region of interest while the corresponding SERS spectra are recorded simultaneously. A detailed comparison between the Raman enhancement and the enhancement caused by the LPR can be performed this way. Another advantage of plasmonic dipole antennas is that they only exhibit one single 
LPR mode, the dipole mode when their arm length remains short enough to exclude higher order resonances. In this case, the Rayleigh scattering spectrum of the nanoantenna is similar to a Lorentzian one because it can be treated as a lossy dipole emitter. This Lorentzian line shape is sensitive to the defects, e.g., the tilt of the side wall introduced by nanofabrication, ${ }^{20}$ which can cause unwanted modes associated with symmetry breaking (Figure S2 in Supporting Information). We shall use this property to control the quality of a large number of nanoantennas by simply checking whether the LPR profile of each antenna is Lorentzian.

Different from previous reports, in this work we focus on the relative enhancement of different Raman modes, instead of monitoring the absolute intensity of individual modes. This strategy not only allows us to investigate the extent to which the excitation of LPR can modify the spectral profile of SERS but also renders the investigation of the EM mechanism more accurate. Indeed, in the perspective of the EM mechanism, the Raman enhancement $g_{\text {em }}$ is the product of two terms:

$$
g_{\text {em }}=\frac{\left|E_{\text {loc }}\left(v_{\text {laser }}\right)\right|^{2}}{\left|E_{\text {inc }}\left(v_{\text {laser }}\right)\right|^{2}} \cdot \frac{\left|E_{\text {loc }}\left(v_{\text {Raman }}\right)\right|^{2}}{\left|E_{\text {inc }}\left(\nu_{\text {Raman }}\right)\right|^{2}}
$$

where, $E$ is electric field intensity, $\left|E_{\text {loc }}\left(\nu_{\text {laser }}\right)\right|^{2} /\left|E_{\text {inc }}\left(\nu_{\text {laser }}\right)\right|^{2}$ represents the enhancement of the local intensity of the incident light at the laser frequency $v_{\text {laser }}$ and $\left|E_{l o c}\left(\nu_{\text {Raman }}\right)\right|^{2} /$ $\left|E_{\text {inc }}\left(v_{\text {Raman }}\right)\right|^{2}$ describes the enhancement of the emission efficiency of the Raman signal at frequency $v_{\text {Raman }}$ caused by the presence of the optical antenna. ${ }^{21}$ In practice, $v_{\text {laser }}$ is a fixed value, and $v_{\text {Raman }}$ varies for different vibrational modes. Hence, a direct verification of the EM mechanism can be performed by evaluating the relative intensity between different modes. Recently, a similar strategy has been reported by Jackson and Halas who investigated how the relative enhancements of different Raman modes are influenced by the geometrical parameters of nanoshell substrates. ${ }^{22}$ In the literature, the Raman enhancement is usually simplified in the form of

$$
g_{\text {em }}=\left|\frac{E_{\text {loc }}\left(\nu_{\text {laser }}\right)}{E_{\text {inc }}\left(v_{\text {laser }}\right)}\right| 4
$$

because the frequency difference between the laser and Raman modes is relatively small compared to the width of the LPR. However, in this work we will show that this approximation is inappropriate and may cause an $\sim 10 \times$ error in the Raman signal. Besides these theoretical considerations, using the relative intensity between different modes can also help us to circumvent practical difficulties, such as estimating the number of molecules and the intrinsic Raman scattering cross-section of the analyte, which often cause inaccuracies in a quantitative analysis. It is worth mentioning that, as a vector field, the polarization of the electric field can also influence the EM enhancement of Raman scattering. ${ }^{21}$ However, this can be ignored, and eq 1 remains appropriate for plasmonic dipole antennas because only the component of the electric field along the antenna can be highly enhanced in their feeding gaps, i.e., the EM mechanism of SERS degenerates into a scalar theory in this case.

The plasmonic dipole antennas were designed with the help of Green's tensor technique, in such a way that the LPR can cover both Raman modes (at $\lambda \cong 650 \mathrm{~nm}$ and $\lambda \cong 700 \mathrm{~nm}$ ) relevant to the experiment. ${ }^{17} \mathrm{Au}$ was used as material for the antennas. Two-dimensional (2D) arrays of antennas with dif-
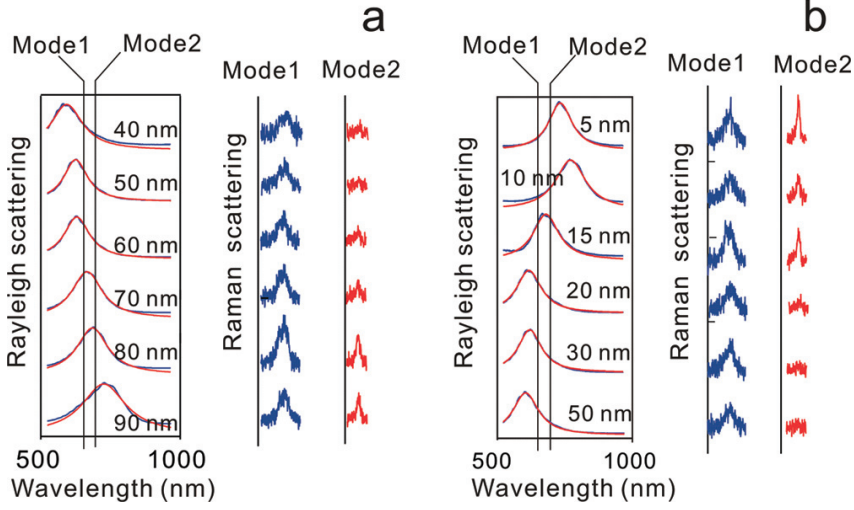

Figure 2. Rayleigh scattering spectra and two surface-enhanced Raman modes of methylene blue molecules measured on the same single plasmonic dipole antennas. Spectra from dipole antennas with different arm lengths and a fixed gap size of $30 \mathrm{~nm}$ are shown in panel a. Panel b displays the spectra from the same single dipole antennas with a fixed arm length of $50 \mathrm{~nm}$ and different gap sizes. The wavelengths of the two different Raman modes are indicated by the two lines in the Rayleigh scattering spectra. The blue and red curves are the experimental data and the Lorentzian fits, respectively.

ferent geometries were fabricated using electron beam lithography with a precision of $10 \mathrm{~nm}$ as shown in Figure S1 (Supporting Information). The thickness of each antenna was $40 \mathrm{~nm}$. The arm length was varied between 40 and $100 \mathrm{~nm}$ in $10 \mathrm{~nm}$ steps along one direction of the array, while the gap width was varied between 5 and $55 \mathrm{~nm}$ in $5 \mathrm{~nm}$ steps along the other direction. The distance between two neighboring antennas was $3 \mu \mathrm{m}$, about 5 times larger than their resonant wavelength, ensuring that there is no cross-talk between neighboring antennas. Dye molecules, brilliant cresyl blue (BCB) or methylene blue, were spin-coated on the structures as Raman probes.

A home-built dark-field microscopy/spectroscopy setup was used to characterize the LPR of each single antenna (Figure S1 in Supporting Information). As aforementioned, all of the Rayleigh scattering spectra were fitted with Lorenzian curves for the quality control of the nanoantennas. The fitting also provides a way to automatically extract the resonance frequency and width of the LPR for each antenna.

As shown in Figure 2a, the LPR of the antennas exhibits a linear dependence of the resonance wavelength on the antenna length, consistent with simulation results reported previously. ${ }^{17}$ Red shifts are also observed when the gap size decreases, as shown in Figure 2b. Interestingly, when the gap size changes from 15 to $10 \mathrm{~nm}$, the spectrum shows a sudden spectral jump over $100 \mathrm{~nm}$. This can be explained by the fact that the resonance frequency is very sensitive to the gap size when the gap is smaller than $10 \mathrm{~nm} \cdot{ }^{17,23}$ Since the precision of electron beam lithography is limited to about $10 \mathrm{~nm}$, fabrication inaccuracy can certainly produce large spectral variations when the gap size is designed below $15 \mathrm{~nm}$.

SERS measurements on the single antennas were performed with a commercial laser scanning confocal Raman microscope (NTEGRA-Spectra, NT-MDT) (Supporting Information). The $632.8 \mathrm{~nm}$ line of a HeNe laser was used as excitation source, and a waveplate was placed in the illumination part to tune the polarization along the antennas. During the measurement, the power reaching the sample was $\sim 2 \mathrm{~mW}$ or $\sim 200 \mathrm{~mW}$ depending on the sample. The collection time for each spectrum was $1 \mathrm{~s}$.

With the help of the markers on the side of the antenna arrays, we were able to identify the antennas unambiguously and consequently perform the Raman measurement on the single 



Figure 3. Spectral map of a 2D antenna array. Laser scanning confocal Raman mapping was performed over an antenna array. (a) The full Raman spectrum of the analyte (BCB) was collected at each pixel of the map. The net intensity of two Raman modes was calculated by integrating the signal covered by the green and red bars after background subtraction (the green and red dashed base-lines are determined with linear fits). (b) The intensity of the two modes was then plotted in one image using the green and red color channels, respectively. Therefore, the color changes from green to yellow and then to red due to the mixing of the two colors when the intensity ratio between mode 2 and mode 1 increases. The periodic bright spots indicate the antennas. (c) Intensity ratio of the two Raman modes on each antenna. (d) Measured resonance wavelength obtained from Rayleigh scattering spectra for the same antennas. (e) Prediction of the relative intensity of the two Raman modes using eq 1 based on the Rayleigh scattering spectra. In $b-e$, the red crosses indicate antennas with a non-Lorentzian LPR line shape.

antennas which had been previously characterized with Rayleigh scattering spectroscopy. We focus on the two dominant modes of the analyte which are at $480 \mathrm{~cm}^{-1}$ and $1515 \mathrm{~cm}^{-1}$ in the case of methylene blue $\left(570 \mathrm{~cm}^{-1}\right.$ and $1655 \mathrm{~cm}^{-1}$ in the case of BCB). A significant change of the relative intensity of these two modes was observed when the resonance frequency of the antenna was tuned over the frequency of those two modes as shown in Figure 2a. When the arm length of the antenna is $40 \mathrm{~nm}$, its resonance frequency is on the blue side of the two modes, and mode 2 is almost invisible; when the antenna arm length increases, the LPR frequency red-shifts, and the intensity of mode 2 increases significantly until it becomes as strong as mode 1. Similar results can be observed when the antenna gap size decreases as shown in Figure $2 \mathrm{~b}$. When the gap size is large, the resonance wavelength is bluer than mode 1 , and the intensity of mode 2 is much lower than that of mode 1 ; when the gap size decreases, the LPR red-shifts, and as a consequence, mode 2 becomes stronger. During this procedure, the relative intensity between the modes 1 and 2 changes by a factor of 8.1 , almost 1 order of magnitude.

The full spectral mapping of both Rayleigh scattering and Raman scattering were collected on 6 different 2D antenna arrays to check whether this dramatic relative enhancement variation is purely caused by the EM mechanism of SERS. Driven by the dipole resonant mode, the near-field enhancement spectrum in the feeding gap of a dipole antenna shares a similar spectral profile with the scattering spectrum of the antenna. Hence, the relative enhancement of the two Raman modes can be predicted using the Rayleigh scattering spectra of the antenna. We calculated the relative Raman enhancement on 6 antenna arrays ( 2 of them were stained with $\mathrm{BCB}$, and the rest was stained with methylene blue) using the Rayleigh scattering data and compared the result with the real Raman measurements (Supporting Information). A correlation coefficient greater than 0.9 was found from 4 out of the 6 antenna arrays, while the correlation coefficients of the rest of the 2 arrays were $\sim 0.8$; Figure 3 shows one typical result. This result not only demonstrates that the LPR can introduce a significant modification of the spectral profile of SERS but also provides a direct validation of the EM mechanism of SERS on single plasmonic antennas. To the best of our knowledge, this is the first time that the EM mechanism of SERS is quantitatively validated on single nanostructures. It shows that eq 2, the commonly used approximation for the EM mechanism of SERS, may cause an error up to 1 order of magnitude and also implies that we can selectively enhance different Raman modes in SERS by tuning the LPR frequency.

Moreover, this dramatic LPR-induced Raman spectral modification provides a possible explanation for some unanswered questions related to single-molecule SERS, such as the low yield of hot spots and the poor reproducibility of SERS spectra in the hot spots. $^{12}$ It was reported that hot spots are rare in aggregated colloids, although these structures have a large number of nanojunctions. Our data indicate that this phenomenon can be understood by the extreme sensitivity of the LPR wavelength to the gap size below a few nanometers. ${ }^{17,23,24}$
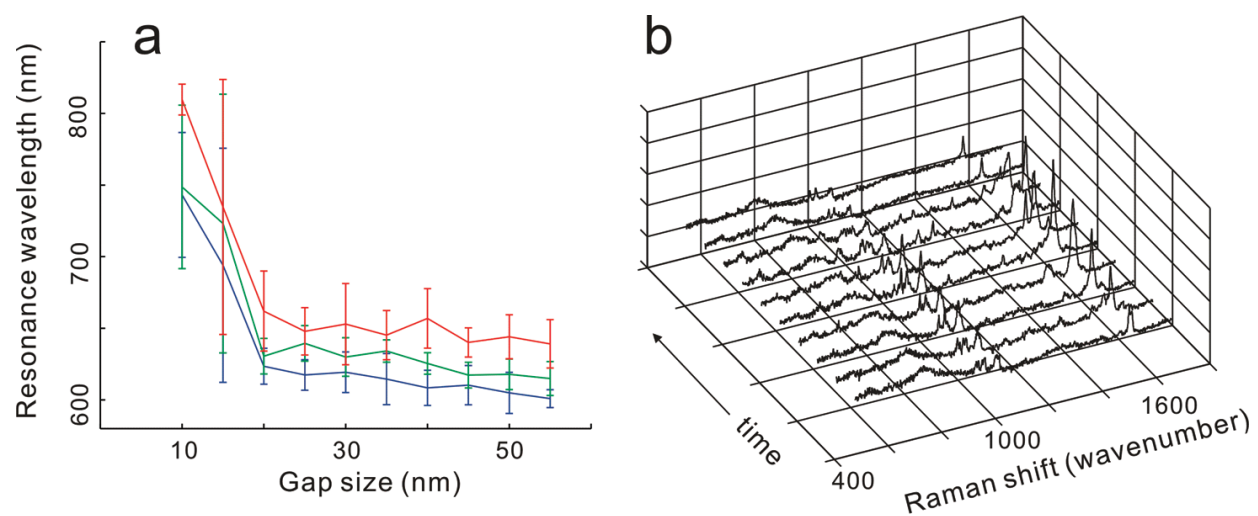

Figure 4. (a) LPR wavelength measured as a function of the antenna gap size. The blue, green, and red curves represent the resonance wavelengths (error bars: s. d. from 6 measurements) of dipole antennas with arm lengths of 40, 50, and $60 \mathrm{~nm}$, respectively. (b) Around 10\% of the antennas with a gap of 10 or $15 \mathrm{~nm}$ show temporal spectroscopic fluctuations. 
In the Rayleigh scattering measurements, dramatic red shifts are observed for the antennas with a designed gap size smaller than $20 \mathrm{~nm}$. Figure 2B shows one example: when the designed gap size decreases from $20 \mathrm{~nm}$ to $15 \mathrm{~nm}$ or from 15 to $10 \mathrm{~nm}$, there is a sudden resonance wavelength jump of more than 50 $\mathrm{nm}$; on the contrary, the resonance shift is less than $30 \mathrm{~nm}$ when the gap size increases from 20 to $50 \mathrm{~nm}$. In order to further evaluate this instability of LPR frequency caused by narrow gaps, we measured the scattering spectra of single antennas from 6 arrays. The results clearly demonstrate the huge LPR shift induced by the gap size, as shown in Figure 4A. The standard deviation of the resonance wavelength for antennas with a designed gap of $10 \mathrm{~nm}$ is greater than $80 \mathrm{~nm}$, while the standard deviation for antennas with a gap greater than $20 \mathrm{~nm}$ is only $15 \mathrm{~nm}$. At the same time, when the gap size decreases from 20 to $15 \mathrm{~nm}$, or from 15 to $10 \mathrm{~nm}$, the resonance shifts are greater than $50 \mathrm{~nm}$. Interestingly, it has been pointed out that this high sensitivity of LPR wavelength to the gap size is a common feature for particle pair structures. ${ }^{23,24}$

Considering that the LPR introduces relative intensity changes of the different vibrational modes, this ultrasensitive gap-sizedependent shift can cause a variation of 1 order of magnitude for the relative enhancement. This provides an explanation for the variety of spectral profiles observed in different singlemolecule SERS measurements.

For the absolute enhancement factor of a single Raman mode, the effect caused by this gap induced LPR jump is even greater. Considering the situation where the LPR wavelength scans from on-resonance to off-resonance, the local field enhancement in the gap can easily change by more than 1 order of magnitude, ${ }^{17}$ and consequently, the variation of the Raman emission enhancement can be greater than 2 orders of magnitude, according to the EM mechanism.

In order to verify this idea, we measured the SERS response from more than 200 single antennas with a narrow gap (i.e., the gap size was designed to be smaller than $20 \mathrm{~nm}$ ). Benzenethiol molecules were used as Raman probes in this measurement because they form a monolayer on $\mathrm{Au}$ surfaces (Supporting Information), and consequently, the number of benzenethiol molecules adsorbed in the gap of each plasmonic dipole antenna is approximately the same. Only around $10 \%$ of those antennas exhibited temporal spectral fluctuations, a phenomenon usually used as an indicator for hot spots, as shown in Figure 4b. This is consistent with the expectation from the Rayleigh scattering measurements.

Let us note that the above-mentioned LPR-driven modeselective SERS mechanism will be more significant when an infrared laser is used instead of a visible source for the following two reasons. First, the wavelength difference between different Raman modes is greater when the excitation wavelength is longer. Second, the better conductivity of metals in the infrared regime produces a narrower LPR width when the resonance wavelength increases. For the same reasons, the LPR introduced spectral variation will also be more significant when Ag is used instead $\mathrm{Au}$ since the conductivity of $\mathrm{Ag}$ is better than that of $\mathrm{Au}$ in the optical regime.

In summary, we have demonstrated mode-selective Raman enhancement using single plasmonic dipole antennas. Experiments show that the relative enhancement of two Raman modes can be selectively modified by almost 1 order of magnitude when tuning the LPR over these two modes, even though their wavelength difference is much smaller than the line width of the LPR. This observation directly points out the limitation of the $g_{\text {em }}=\left|E_{\text {loc }}\left(v_{\text {laser }}\right) / E_{0}\left(v_{\text {laser }}\right)\right|^{4}$ approximation, which is widely used in SERS studies. Thanks to this relative enhancement effect, we have furthermore correlated the SERS signal to the Rayleigh scattering spectra from the same single nanoantennas using the EM mechanism of SERS. This provides for the first time a quantitative validation of the EM mechanism of SERS on single nanostructures. Besides, we also found that the LPR wavelength of the nanoantenna is extremely sensitive to the gap size when its dimension is only a few nanometers wide. This phenomenon provides a possible explanation for the low spectral reproducibility of single-molecule SERS experiments.

Acknowledgment. We thank Dr. Boon-Siang Yeo for helpful discussions. Fundings from EPFL STI-Seed Fund, Swiss National Centre of Competence in Research Nanoscale Science and Swiss National Science Foundation, R'EQUIP are gratefully acknowledged.

Supporting Information Available: Experimental and data analysis methods including the sample preparation, the elastic and inelastic scattering spectrum measurements, line shape analysis of the elastic scattering spectra of the plasmonic dipole antennas, and the calculation method of the relative enhancement of the two Raman modes. This material is available free of charge via the Internet at http://pubs.acs.org.

\section{References and Notes}

(1) Jeanmaire, D. L.; van Duyne, R. P. J. Electroanal. Chem. 1977, $84,1-20$.

(2) Kneipp, K.; et al. Phys. Rev. Lett. 1997, 78, 1667-1670.

(3) Nie, S. M.; Emory, R. Science 1997, 275, 1102-1106.

(4) Kneipp, K.; Kneipp, H.; Itzkan, I.; Dasari, R. R.; Feld, M. S. Chem. Rev. 1999, 99, 2957-2976.

(5) Stiles, P. L.; Kieringer, J. A.; Shah, N. C.; Van Duyne, R. P. Annu. Rev. Anal. Chem. 2008, 1, 601-626.

(6) Moskovits, M. Rev. Mod. Phys. 1985, 57, 783-826. 1166.

(7) Garcia-Vidal, F. J.; Pendry, J. B. Phys. Rev. Lett. 1996, 77, 1163-

(8) Xu, H. X.; Aizpurua, J.; Kall, M.; Apell, P. Phys. Rev. E 2000, 62, $4318-4324$.

(9) Jiang, J.; Bosnick, K.; Maillard, M.; Brus, L. J. Phys. Chem. B 2003, 107, 9964-9972.

(10) Camden, J. P.; et al. J. Am. Chem. Soc. 2008, 130, 12616-12617.

(11) Lee, S. J.; Guan, Z. Q.; Xu, H. X.; Moskovits, M. J. Phys. Chem. C 2007, 111, 17985-17988.

(12) Doering, W. E.; Nie, S. M. J. Phys. Chem. B 2002, 106, 311-317.

(13) Wang, Z. J.; Rothberg, L. J. J. Phys. Chem. B 2005, 109, 33873391.

(14) Ward, D. R.; et al. Nano Lett. 2008, 8, 919-924.

(15) Otto, A.; Mrozek, I.; Grabhorn, H.; Akemann, W. J. Phys.: Condens. Matter 1992, 4, 1143-1212.

(16) Muhlschlegel, P.; Eisler, H. J.; Martin, J. F. O.; Hecht, B.; Pohl, D. W. Science 2005, 308, 1607-1609.

(17) Fischer, H.; Martin, J. F. O. Opt. Express 2008, 16, 9144-9154.

(18) Taminiau, T. H.; Stefani, F. D.; Segerink, F. B.; Van Hulst, N. F. Nat. Photonics 2008, 2, 234-237.

(19) Haynes, C. L.; Van Duyne, R. P. J. Phys. Chem. B 2003, 107, $7426-7433$

(20) Merlein, J.; et al. Nat. Photonics 2008, 2, 230-233.

(21) Le Ru, E. C.; Etchegoin, P. G. Chem. Phys. Lett. 2006, 423, 6366.

(22) Jackson, J. B.; Halas, N. J. Proc. Natl. Acad. Sci. U.S.A. 2004, 101, 17930-17935.

(23) Jain, P. K.; Huang, W. Y.; El-Sayed, M. A. Nano Lett. 2007, 7, 2080-2088.

(24) Lassiter, J. B.; et al. Nano Lett. 2008, 8, 1212-1218.

JP9042304 